\title{
Two-level finite element method with a stabilizing subgrid for the incompressible MHD equations
}

\author{
S. H. Aydin ${ }^{1, *, \dagger}$, A. I. Nesliturk ${ }^{1,2}$ and M. Tezer-Sezgin ${ }^{1,3}$ \\ ${ }^{1}$ Institute of Applied Mathematics, Middle East Technical University, 06531 Ankara, Turkey \\ ${ }^{2}$ Izmir Institute of Technology, Department of Mathematics, 35430 Izmir, Turkey \\ ${ }^{3}$ Department of Mathematics, Middle East Technical University, 06531 Ankara, Turkey
}

\begin{abstract}
SUMMARY
We consider the Galerkin finite element method (FEM) for the incompressible magnetohydrodynamic (MHD) equations in two dimension. The domain is discretized into a set of regular triangular elements and the finite-dimensional spaces employed consist of piecewise continuous linear interpolants enriched with the residual-free bubble functions. To find the bubble part of the solution, a two-level FEM with a stabilizing subgrid of a single node is described and its application to the MHD equations is displayed. Numerical approximations employing the proposed algorithm are presented for three benchmark problems including the MHD cavity flow and the MHD flow over a step. The results show that the proper choice of the subgrid node is crucial to get stable and accurate numerical approximations consistent with the physical configuration of the problem at a cheap computational cost. Furthermore, the approximate solutions obtained show the well-known characteristics of the MHD flow. Copyright (C) 2009 John Wiley \& Sons, Ltd.
\end{abstract}

Received 3 July 2008; Revised 19 January 2009; Accepted 19 January 2009

KEY WORDS: stabilizing subgrid; MHD equations; two-level finite element method

\section{INTRODUCTION}

Magnetohydrodynamics (MHD) is the theory of the macroscopic interaction of electrically conducting fluid and electromagnetic fields. Applications arise in astronomy and geophysics

\footnotetext{
*Correspondence to: S. H. Aydın, Institute of Applied Mathematics, Middle East Technical University, 06531 Ankara, Turkey.

†E-mail: saydin@metu.edu.tr

Contract/grant sponsor: The Scientific and Technical Research Council of Turkey (TUBITAK); contract/grant number: 105T091
} 
as well as in connection with numerous engineering problems, such as liquid-metal cooling of nuclear reactors, electromagnetic casting of metals, MHD power generation and MHD ion propulsion. In MHD problems we deal with a flow of a viscous, incompressible fluid which has the property of electric current conduction and interacting with electromagnetic fields. The conducting fluid can induce electric current and interact with the magnetic field. This interaction in turn produces the Lorentz force on the fluid and can greatly change the fluid behavior. Thus, MHD flow is governed by the Navier-Stokes and pre-Maxwell equations coupled via the Lorentz force. The theory and the mathematical modeling of the MHD flow can be found in $[1,2]$.

A considerable amount of research activity has been devoted to the analysis of numerical methods for the simulation of MHD flows by using finite difference method (FDM). A nine-node grid FD approximation was applied to solving MHD equations inside a channel with a rapidly expanded section, in [3]. A description was made using two vector potentials and the vorticity vector, which form a system of three simultaneous, quasilinear equations of the elliptic type. Sekhar et al. [4] have obtained FD solutions in terms of stream function-vorticity for solving the steady MHD flow past a sphere with an applied magnetic field parallel to the main flow at low and moderate Reynolds number. In the paper written by Shue and Lin [5], a primitive variable approach for solving the magnetic field and hydrodynamic field equations has been given with the alternating direction implicit (ADI) solution algorithm. An extension of the generalized Peaceman and Rachford ADI scheme was presented in [6]. The discretized conservation equations are solved in stream functionvorticity formulation for low magnetic Reynolds number. 3-D numerical calculations on liquidmetal MHD flow through a rectangular channel in the inlet region have been performed by Kumamaru et al. [7] using the FDM and following the MAC method.

On the other hand, most of the numerical solutions of the MHD equations are performed by using the finite element method (FEM) because it is more appropriate for a wide class of problem configurations. The existence of solutions of both continuous and discrete MHD problems without any condition on the boundary data of the velocity was derived in [8], and Gunzburger et al. [9] have shown that the existence and uniqueness of the solution of a weak formulation of the MHD equations can be guaranteed. Meir and Schmidt [10] and Schötzau [11] have carried out error analysis on their finite element solutions of MHD problems and established optimal-order error bounds. Mixed FE approximation of incompressible MHD problems based on weighted regularization has been analyzed by Hasler et al. [12]. Well-posedness of this approach, the existence and uniqueness of the results, quasi-optimal error bounds were also provided. By using a two-level FEM (TLFEM) for discretizing the stationary MHD equations, Layton et al. [13] have proved well-posedness of their algorithm and gave optimal error bounds. In their study, they have solved the nonlinear problem first on a coarse mesh and then the linear one on a fine mesh.

The study of MHD duct flows has great relevance for nuclear reactor cooling systems, MHD flowmeters and MHD micropumps. Such MHD flows have been studied under the assumption that the flow is fully developed. Then the problem dimensionality reduces from three to two and the application of the governing equations is allowed only in a transversal section of the duct. Verardi and Cardoso [14,15], Krzeminski et al. [16], Tezer-Sezgin and Köksal [17] have given FE solutions of MHD equations in rectangular ducts. A stabilized FEM using the residual-free bubble (RFB) functions has been proposed by Neslitürk and Tezer-Sezgin $[18,19]$ for solving steady MHD duct flow problems at high Hartmann numbers.

It is known that the small hydrodynamic diffusion may induce some well-known numerical instabilities. It is therefore natural to use some stabilization techniques for the solution of MHD 
equations. Gerbeau [20] has carried out a stabilized FEM procedure in terms of velocity $\mathbf{u}$, the pressure $p$ in the fluid and the magnetic field $\mathbf{B}$, including a convergence proof. A stabilization technique is used in order to allow equal-order interpolation on tetrahedral elements of all variables. In the papers by Salah et al. [21,22], Codina and Silva [23], they have presented a stabilized FEM for the full MHD equations by including a magnetic pressure as unknowns to enforce the divergence-free condition for the numerical approximation of the magnetic field.

In this study, we deal with the numerical solution of the incompressible MHD equations by a TLFEM using a stabilizing subgrid. The unknowns in the equations are the primitive variables as the velocity $\mathbf{u}$, the magnetic field $\mathbf{B}$ and the pressure in the fluid $p$. It is known that, besides the high values of problem parameters (fluid Reynolds number Re, magnetic Reynolds number Rem and the Hartmann number $\mathrm{Ha}$ ), the coupling of the equations produces numerical instabilities. Therefore, a stabilized formulation should be considered in calculating the approximate solution of the problem.

We propose a stabilizing subgrid method (SSM) for the approximate solution of the incompressible MHD equations, which is based on the proper choice of the subgrid point inside each element in a triangular discretization of the domain. The method has been first proposed for the convection-diffusion problem [24] and extended to the Navier-Stokes equations in [25]. The velocity and the magnetic field spaces are enriched by the bubble functions that are added to continuous piecewise-linear function spaces. The function space for the pressure is selected as only the continuous piecewise linears. As a result, a new formulation similar to the streamline-upwind Petrov-Galerkin (SUPG)-[20] type stabilized formulation of the MHD equations is obtained. The nonlinearity emanating from the nature of the equations is treated through iterations. Numerical experiments show that the SSM is robust and produces accurate approximations compared with the SUPG method at a cheap computational cost.

We organize the paper as follows. In Section 2, we describe the governing equations of the MHD flow. In Section 3, we present the standard Galerkin formulation of the problem. We recall the basics of the stabilized FEMs in Section 4. In Section 5, we describe a TLFEM using the approximate RBF functions to determine a stabilizing subgrid and display its application to MHD equations. Implementational issues related to the numerical method under investigation will also be discussed in this section. We illustrate the potential of the method in Section 6 by presenting some numerical experiments obtained in different problem configurations. Conclusions are drawn in Section 7.

\section{GOVERNING EQUATIONS IN MHD PROBLEM}

MHD equations involve the coupling of hydrodynamic and electromagnetic effects and constitute a differential system for modeling the incompressible conducting fluid motion in a magnetic field [20]. These equations in primary unknown variables $\mathbf{u}, \mathbf{B}$ and $p$ can be given as

$$
\begin{aligned}
\rho \mathbf{u} \cdot \nabla \mathbf{u}-\eta \Delta \mathbf{u}+\nabla p+\frac{1}{\mu} \mathbf{B} \times \operatorname{curl} \mathbf{B} & =\rho \mathbf{f} \\
\operatorname{div} \mathbf{u} & =0
\end{aligned}
$$




$$
\begin{aligned}
\frac{1}{\mu \sigma} \operatorname{curl}(\operatorname{curl} \mathbf{B})-\operatorname{curl}(\mathbf{u} \times \mathbf{B}) & =0 \\
\operatorname{div} \mathbf{B} & =0
\end{aligned}
$$

where $\mathbf{u}=\left(u_{1}, u_{2}\right)$ is the velocity field, $\mathbf{B}=\left(B_{1}, B_{2}\right)$ is the magnetic field and $p$ is the pressure in the fluid, $\mathbf{f}$ denotes the external force, $\rho$ is the density of the fluid, $\eta$ is the viscosity, $\sigma$ is the electrical conductivity and $\mu$ is the magnetic permeability. In order to bring the MHD equations to a non-dimensional form, we introduce a characteristic value $\mathbf{U}_{0}$ for the velocity field, the intensity of the applied magnetic field $\mathbf{B}_{0}$ and a characteristic length $L$. The important non-dimensional parameters in the model are the fluid Reynolds number $(R e)$, the magnetic Reynolds number (Rem), the Hartmann number $(\mathrm{Ha})$ and the Coupling number $(\mathrm{S})$, which are defined as

$$
\begin{gathered}
R e=\frac{\rho \mathbf{U}_{0} L}{\eta} \\
\operatorname{Rem}=\mu \sigma \mathbf{U}_{0} L \\
S=\frac{\mathbf{B}_{0}^{2}}{\mu \rho \mathbf{U}_{0}} \\
H a=\sqrt{\operatorname{ReRem} S}
\end{gathered}
$$

In addition, $\mathbf{u}=\tilde{\mathbf{u}} / \mathbf{U}_{0}, \mathbf{B}=\tilde{\mathbf{B}} / \mathbf{B}_{0}, p=\tilde{p} / \rho \mathbf{U}_{0}^{2}$ and $\mathbf{f}=\tilde{\mathbf{f}} L / \mathbf{U}_{0}^{2}$ are made use of for the physical quantities $\tilde{\mathbf{u}}, \tilde{\mathbf{B}}, \tilde{p}$ and $\tilde{\mathbf{f}}$ in the non-dimensionalizing. Then MHD equations in non-dimensional form are expressed as

$$
\begin{gathered}
\mathbf{u} \cdot \nabla \mathbf{u}-\frac{1}{\operatorname{Re}} \Delta \mathbf{u}+\nabla p-\frac{H a^{2}}{\operatorname{Re} \cdot \operatorname{Rem}}(\nabla \times \mathbf{B}) \times \mathbf{B}=\mathbf{f} \\
\nabla \cdot \mathbf{u}=0 \\
-\nabla \times(\mathbf{u} \times \mathbf{B})+\frac{1}{\operatorname{Rem}} \nabla \times(\nabla \times \mathbf{B})=0 \\
\nabla \cdot \mathbf{B}=0
\end{gathered}
$$

By using the following identities:

$$
\begin{gathered}
\nabla \times(\nabla \times \mathbf{B})=-\nabla^{2} \mathbf{B}+\nabla(\nabla \cdot \mathbf{B}) \\
\nabla \cdot \mathbf{B}=0
\end{gathered}
$$

we can transform the MHD equations (2)-(5) into the following explicit form in 2-D:

$$
\begin{aligned}
& u_{1} \frac{\partial u_{1}}{\partial x}+u_{2} \frac{\partial u_{1}}{\partial y}-\frac{1}{R e}\left(\frac{\partial^{2} u_{1}}{\partial x^{2}}+\frac{\partial^{2} u_{1}}{\partial y^{2}}\right)+\frac{\partial p}{\partial x}-S B_{2}\left(\frac{\partial B_{1}}{\partial y}-\frac{\partial B_{2}}{\partial x}\right)=f_{1} \\
& u_{1} \frac{\partial u_{2}}{\partial x}+u_{2} \frac{\partial u_{2}}{\partial y}-\frac{1}{R e}\left(\frac{\partial^{2} u_{2}}{\partial x^{2}}+\frac{\partial^{2} u_{2}}{\partial y^{2}}\right)+\frac{\partial p}{\partial y}+S B_{1}\left(\frac{\partial B_{1}}{\partial y}-\frac{\partial B_{2}}{\partial x}\right)=f_{2}
\end{aligned}
$$




$$
\begin{gathered}
u_{1} \frac{\partial B_{1}}{\partial x}+u_{2} \frac{\partial B_{1}}{\partial y}+\frac{\partial u_{2}}{\partial y} B_{1}-\frac{\partial u_{1}}{\partial y} B_{2}-\frac{1}{\operatorname{Rem}}\left(\frac{\partial^{2} B_{1}}{\partial x^{2}}+\frac{\partial^{2} B_{1}}{\partial y^{2}}\right)=0 \\
u_{1} \frac{\partial B_{2}}{\partial x}+u_{2} \frac{\partial B_{2}}{\partial y}+\frac{\partial u_{1}}{\partial x} B_{2}-\frac{\partial u_{2}}{\partial x} B_{1}-\frac{1}{\operatorname{Rem}}\left(\frac{\partial^{2} B_{2}}{\partial x^{2}}+\frac{\partial^{2} B_{2}}{\partial y^{2}}\right)=0 \\
\frac{\partial u_{1}}{\partial x}+\frac{\partial u_{2}}{\partial y}=0
\end{gathered}
$$

Before starting to present the numerical methods, we set the notation. We use standard notation for function spaces: $C^{0}(\bar{\Omega})$ is the space of continuous functions on the closure of $\Omega, L^{2}(\Omega)$ is the space of square integrable functions over the domain $\Omega, L_{0}^{2}(\Omega)$ is the space of $L^{2}(\Omega)$ functions with zero mean over $\Omega, H^{1}(\Omega)$ is the Sobolev space of $L^{2}(\Omega)$ functions whose derivatives are square integrable functions in $\Omega$ and $H_{0}^{1}(\Omega)$ is the Sobolev subspace of $H^{1}(\Omega)$ functions in $\Omega$ with zero value on the boundary $\partial \Omega$.

\section{THE STANDARD FEM FOR MHD EQUATIONS}

Consider the incompressible MHD equation in a bounded domain $\Omega \in \mathbb{R}^{2}$

$$
\begin{aligned}
& \mathbf{u} \cdot \nabla \mathbf{u}-\frac{1}{R e} \Delta \mathbf{u}+\nabla p-\frac{H a^{2}}{R e \cdot R e m}(\nabla \times \mathbf{B}) \times \mathbf{B}=\mathbf{f} \\
& -\nabla \times(\mathbf{u} \times \mathbf{B})-\frac{1}{\operatorname{Rem}} \Delta \mathbf{B}=0 \\
& \nabla \cdot \mathbf{u}=0
\end{aligned}
$$

with the appropriate boundary conditions. Then the weak formulation of the problem (11) can be stated as: Find $\mathbf{u} \in V=H_{0}^{1}(\Omega)^{2}, \mathbf{B} \in M=H^{1}(\Omega)^{2}$ and $p \in W=L_{0}^{2}(\Omega)$ such that

$$
B(\mathbf{u}, \mathbf{B}, p ; \mathbf{v}, \mathbf{C}, q)=(\mathbf{f}, \mathbf{v}) \quad \forall(\mathbf{v}, \mathbf{C}, q) \in(V, M, W)
$$

where

$$
\begin{aligned}
B(\mathbf{u}, \mathbf{B}, p ; \mathbf{v}, \mathbf{C}, q)= & ((\nabla \mathbf{u}) \mathbf{u}, \mathbf{v})+\frac{1}{R e}(\nabla \mathbf{u}, \nabla \mathbf{v})-(p, \nabla \mathbf{v})-S((\nabla \times \mathbf{B}) \times \mathbf{B}, \mathbf{v}) \\
& -(\nabla \times(\mathbf{u} \times \mathbf{B}), \mathbf{C})+\frac{1}{\operatorname{Rem}}(\nabla \mathbf{B}, \nabla \mathbf{C})+(q, \nabla \cdot \mathbf{u})
\end{aligned}
$$

To introduce a FEM, we begin by partitioning the domain into triangular elements in a standard way (e.g. no overlapping, no vertex on the edge of a neighboring elements, etc.) and let $\Omega_{h}$ be such a partition of $\Omega$. We define

$$
X_{h}=\left\{v_{h} \in C^{0}(\bar{\Omega}),\left.v_{h}\right|_{K} \in P_{k}(K), \forall K \in \Omega_{h}\right\}
$$

where $P_{k}(K)$ correspond to the space of $k$ th-order polynomial shape functions over triangular elements. The finite element spaces employed are as follows: For the velocity component 
$\mathbf{u} ; V_{h}=\left(X_{h} \cap H_{0}^{1}(\Omega)\right)^{2}$, for the magnetic field $\mathbf{B} ; M_{h}=\left(X_{h}\right)^{2} \cap H^{1}(\Omega)^{2}$ and for the pressure $p ; W_{h}=X_{h} \cap L_{0}^{2}(\Omega)$. Now the Galerkin finite element formulation of the problems reads: find $\left(\mathbf{u}_{h}, \mathbf{B}_{h}, p_{h}\right) \in\left(V_{h}, M_{h}, W_{h}\right)$ such that

$$
B_{G}\left(\mathbf{u}_{h}, \mathbf{B}_{h}, p_{h} ; \mathbf{v}_{h}, \mathbf{C}_{h}, q_{h}\right)=\left(\mathbf{f}, \mathbf{v}_{h}\right) \quad \forall\left(\mathbf{v}_{h}, \mathbf{C}_{h}, q_{h}\right) \in\left(V_{h}, M_{h}, W_{h}\right)
$$

where

$$
\begin{aligned}
B_{G}\left(\mathbf{u}_{h}, \mathbf{B}_{h}, p_{h} ; \mathbf{v}_{h}, \mathbf{C}_{h}, q_{h}\right)= & \left(\left(\nabla \mathbf{u}_{h}\right) \mathbf{u}_{h}, \mathbf{v}_{h}\right)+\frac{1}{R e}\left(\nabla \mathbf{u}_{h}, \nabla \mathbf{v}_{h}\right)-\left(p_{h}, \nabla \mathbf{v}_{h}\right)-S\left(\left(\nabla \times \mathbf{B}_{h}\right) \times \mathbf{B}_{h}, \mathbf{v}_{h}\right) \\
& -\left(\nabla \times\left(\mathbf{u}_{h} \times \mathbf{B}_{h}\right), \mathbf{C}_{h}\right)+\frac{1}{\operatorname{Rem}}\left(\nabla \mathbf{B}_{h}, \nabla \mathbf{C}_{h}\right)+\left(q_{h}, \nabla \cdot \mathbf{u}_{h}\right)
\end{aligned}
$$

We carry out a simple iterative procedure to linearize the nonlinear terms in (13)

$$
\mathbf{u}^{n+1}=\mathbf{u}^{n}+\hat{\mathbf{u}} \quad \text { and } \quad \mathbf{B}^{n+1}=\mathbf{B}^{n}+\hat{\mathbf{B}}
$$

where $\mathbf{u}^{n}, \mathbf{B}^{n}$ and $\hat{\mathbf{u}}, \hat{\mathbf{B}}$ are the previous iteration and correction values of the velocity and the magnetic field, respectively. Thus, we make the following approximations as:

$$
\begin{gathered}
\left(\nabla \mathbf{u}^{n+1}\right) \mathbf{u}^{n+1} \approx\left(\nabla \mathbf{u}^{n}\right) \mathbf{u}^{n}+\left(\nabla \mathbf{u}^{n}\right) \hat{\mathbf{u}}+(\nabla \hat{\mathbf{u}}) \mathbf{u}^{n} \\
\left(\nabla \times \mathbf{B}^{n+1}\right) \times \mathbf{B}^{n+1} \approx\left(\nabla \times \mathbf{B}^{n}\right) \times \mathbf{B}^{n}+\left(\nabla \times \mathbf{B}^{n}\right) \times \hat{\mathbf{B}}+(\nabla \times \hat{\mathbf{B}}) \times \mathbf{B}^{n} \\
\nabla \times\left(\mathbf{u}^{n+1} \times \mathbf{B}^{n+1}\right) \approx \nabla \times\left(\mathbf{u}^{n} \times \mathbf{B}^{n}\right)+\nabla \times\left(\mathbf{u}^{n} \times \hat{\mathbf{B}}\right)+\nabla \times\left(\hat{\mathbf{u}} \times \mathbf{B}^{n}\right)
\end{gathered}
$$

\section{THE SUPG METHOD FOR MHD EQUATIONS}

It is known that the existence of the pressure term in the MHD equations brings some oscillations to the numerical solution when the standard Galerkin FEM is employed. In order to eliminate these numerical difficulties, an appropriate pair of finite element spaces satisfying Babuska-Brezzi condition must be used [26,27]. A possible choice is to use quadratic shape functions for both the velocity and the magnetic field variables and linear shape functions for the pressure $\left(Q_{2}-\right.$ $Q_{2}-Q 1$ elements). However, the Babuska-Brezzi condition does not allow the use of equal-order interpolations that are the most favorable choice from the computational point of view. Another possibility to get rid of unphysical oscillations in the numerical solution is to use stabilized FEMs in the solution procedure that allows to use equal-order shape functions. One of the most favorite class of stabilized formulations goes under the name SUPG $[28,29]$ and the application of one of its variants to the MHD equations using linear elements is given in [20]: Find $\left(\mathbf{u}_{1}, \mathbf{B}_{1}, p_{1}\right) \in$ $\left(V_{1}, M_{1}, W_{1}\right)$ such that

$$
\begin{aligned}
& B_{G}\left(\mathbf{u}_{1}, \mathbf{B}_{1}, p_{1} ; \mathbf{v}_{1}, \mathbf{C}_{1}, q_{1}\right)+B_{\mathrm{SUPG}}\left(\mathbf{u}_{1}, \mathbf{B}_{1}, p_{1} ; \mathbf{v}_{1}, \mathbf{C}_{1}, q_{1}\right) \\
& \quad=\left(\mathbf{f}, \mathbf{v}_{1}\right)+F_{\mathrm{SUPG}}\left(\mathbf{v}_{1}, \mathbf{C}_{1}, q_{1}\right) \quad \forall\left(\mathbf{v}_{1}, \mathbf{C}_{1}, q_{1}\right) \in\left(V_{1}, M_{1}, W_{1}\right)
\end{aligned}
$$


where

$$
\begin{aligned}
& B_{\mathrm{SUPG}}\left(\mathbf{u}_{1}, \mathbf{B}_{1}, p_{1} ; \mathbf{v}_{1}, \mathbf{C}_{1}, q_{1}\right) \\
& =\sum_{K \in \Omega_{h}} \tau_{u}^{\mathrm{SUPG}}\left(\left(\nabla \mathbf{u}_{1}\right) \mathbf{u}_{1}+\nabla p_{1}+S \mathbf{B}_{1} \times\left(\nabla \times \mathbf{B}_{1}\right),\left(\nabla \mathbf{v}_{1}\right) \mathbf{u}_{1}+\nabla q_{1}+S \mathbf{B}_{1} \times\left(\nabla \times \mathbf{C}_{1}\right)\right) \\
& \quad+\sum_{K \in \Omega_{h}} \tau_{B}^{\mathrm{SUPG}}\left(-\nabla \times\left(\mathbf{u}_{1} \times \mathbf{B}_{1}\right),-\nabla \times\left(\mathbf{v}_{1} \times \mathbf{B}_{1}\right)\right) \\
& \quad F_{\mathrm{SUPG}}\left(\mathbf{v}_{1}, \mathbf{C}_{1}, q_{1}\right)=\sum_{K \in \Omega_{h}} \tau_{u}^{\mathrm{SUPG}}\left(\mathbf{f},\left(\nabla \mathbf{v}_{1}\right) \mathbf{u}_{1}+\nabla q_{1}+S \mathbf{B}_{1} \times\left(\nabla \times \mathbf{C}_{1}\right)\right)
\end{aligned}
$$

with the stabilization parameters

$$
\tau_{u}^{\mathrm{SUPG}}=\left\{\begin{array}{ll}
\frac{h_{K}}{2\left|\mathbf{u}_{1}^{n}\right|_{K}} & \text { if } P e_{k} \geqslant 1 \\
\frac{h_{K}^{2}}{12 \varepsilon} & \text { if } P e_{k}<1
\end{array} \quad \text { and } \quad \tau_{B}^{\mathrm{SUPG}}=\frac{h_{K}^{2}}{12 \beta}\right.
$$

where $h_{K}$ is the diameter of the element $K, P e_{K}=\left|\mathbf{u}_{1}^{n}\right|_{K} h_{K} / 6 \varepsilon$ is the Peclet number, $\varepsilon=1 / R e$ and $\beta=1 /$ Rem.

The SUPG method is computationally cheap and practically easy to implement. Although it is a very effective way of obtaining numerically stable solutions, the fact that it gives too much diffusion to the system that causes the method to receive critics.

\section{THE STABILIZING SUBGRID METHOD (SSM)}

In this section, a more accurate stabilized formulation, the SSM is presented for the solution of the MHD equations. The key idea is to enrich the finite element spaces with some appropriate functions so that the resulting numerical method gives rise to a stabilized formulation without increasing the size of stiffness matrix. In that context, the finite element approximation spaces for the velocity and the magnetic field are enriched by using bubble functions

$$
V_{h}=V_{1} \oplus V_{B} \quad \text { and } \quad M_{h}=M_{1} \oplus M_{B}
$$

where $V_{1}$ and $M_{1}$ are the linear interpolation spaces, $V_{B}$ and $M_{B}$ are the bubble function spaces whose elements vanish on the element boundary. We choose the bubble functions we employ in a special manner such that $\mathbf{u}_{b} \in V_{B}$ and $\mathbf{B}_{b} \in M_{B}$ satisfy the following equations inside each $K$ :

$$
\left.\left.\begin{array}{r}
\left(\nabla\left(\mathbf{u}_{1}^{n+1}+\mathbf{u}_{b}^{n+1}\right)\right) \mathbf{u}_{1}^{n}-\varepsilon \Delta\left(\mathbf{u}_{1}^{n+1}+\mathbf{u}_{b}^{n+1}\right)+\nabla p_{1}^{n+1}-S\left(\nabla \times \mathbf{B}_{1}^{n+1}\right) \times \mathbf{B}_{1}^{n}=\mathbf{f} \\
-\nabla \times\left(\mathbf{u}_{1}^{n} \times \mathbf{B}_{1}^{n+1}\right)-\beta \Delta\left(\mathbf{B}_{1}^{n+1}+\mathbf{B}_{b}^{n+1}\right)=0
\end{array}\right\} \quad \begin{array}{r}
\text { in } K \\
\mathbf{u}_{b}^{n+1}=0 \\
\mathbf{B}_{b}^{n+1}=0
\end{array}\right\} \quad \begin{aligned}
& \text { on } \partial K
\end{aligned}
$$

Then, this special choice of bubble functions in (17), so-called the RFB, enables us to employ the static condensation procedure, from which we obtain the Galerkin formulation using piecewise 
linears for the problem (13) modified by the RFBs [30-34]. Thus, the resulting numerical method reads: Find $\left(\mathbf{u}_{h}, \mathbf{B}_{h}, p_{1}\right) \in\left(V_{h}, M_{h}, W_{1}\right)$ such that

$$
\begin{aligned}
B_{G}\left(\mathbf{u}_{1}, \mathbf{B}_{1}, p_{1} ; \mathbf{v}_{1}, \mathbf{C}_{1}, q_{1}\right)+B_{G}\left(\mathbf{u}_{b}, \mathbf{B}_{b}, p_{1} ; \mathbf{v}_{1}, \mathbf{C}_{1}, q_{1}\right) & =\left(\mathbf{f}, \mathbf{v}_{1}\right) \\
\forall\left(\mathbf{v}_{1}, \mathbf{C}_{1}, q_{1}\right) & \in\left(V_{1}, M_{1}, W_{1}\right)
\end{aligned}
$$

where

$$
\begin{gathered}
B_{G}\left(\mathbf{u}_{1}, \mathbf{B}_{1}, p_{1} ; \mathbf{v}_{1}, \mathbf{C}_{1}, q_{1}\right)=\left(\left(\nabla \mathbf{u}_{1}\right) \mathbf{u}_{1}, \mathbf{v}_{1}\right)+\varepsilon\left(\nabla \mathbf{u}_{1}, \nabla \mathbf{v}_{1}\right)-\left(p_{1}, \nabla \mathbf{v}_{1}\right)-S\left(\left(\nabla \times \mathbf{B}_{1}\right) \times \mathbf{B}_{1}, \mathbf{v}_{1}\right) \\
-\left(\nabla \times\left(\mathbf{u}_{1} \times \mathbf{B}_{1}\right), \mathbf{C}_{1}\right)+\beta\left(\nabla \mathbf{B}_{1}, \nabla \mathbf{C}_{1}\right)+\left(q_{1}, \nabla \cdot \mathbf{u}_{1}\right) \\
B_{G}\left(\mathbf{u}_{b}, \mathbf{B}_{b}, p_{1} ; \mathbf{v}_{1}, \mathbf{C}_{1}, q_{1}\right)= \\
\left(\left(\nabla \mathbf{u}_{b}\right) \mathbf{u}_{1}, \mathbf{v}_{1}\right)+\varepsilon\left(\nabla \mathbf{u}_{b}, \nabla \mathbf{v}_{1}\right)-S\left(\left(\nabla \times \mathbf{B}_{b}\right) \times \mathbf{B}_{1}, \mathbf{v}_{1}\right) \\
-\left(\nabla \times\left(\mathbf{u}_{b} \times \mathbf{B}_{1}\right), \mathbf{C}_{1}\right)+\beta\left(\nabla \mathbf{B}_{b}, \nabla \mathbf{C}_{1}\right)+\left(q_{1}, \nabla \cdot \mathbf{u}_{b}\right)
\end{gathered}
$$

It can be proved that the elimination of the terms containing bubble functions in (18) gives rise to a formulation similar to a stabilized FEM of the SUPG type. Following the lines of [35], one can prove that

$$
\begin{aligned}
B_{G}\left(\mathbf{u}_{b}, \mathbf{B}_{b}, p_{1} ; \mathbf{v}_{1}, \mathbf{C}_{1}, q_{1}\right)= & \tau_{u}^{\mathrm{RFB}}\left(\left(\nabla \mathbf{u}_{1}\right) \mathbf{u}_{1}+\nabla p_{1}+S \mathbf{B}_{1} \times\left(\nabla \times \mathbf{B}_{1}\right)-\mathbf{f},\left(\nabla \mathbf{v}_{1}\right) \mathbf{u}_{1}+\nabla q_{1}+S \mathbf{B}_{1}\right. \\
& \left.\times\left(\nabla \times \mathbf{C}_{1}\right)\right)+\tau_{B}^{\mathrm{RFB}}\left(\nabla \times\left(\mathbf{u}_{1} \times \mathbf{B}_{1}\right), \nabla \times\left(\mathbf{v}_{1} \times \mathbf{B}_{1}\right)\right)
\end{aligned}
$$

These additional terms make the numerical method to recover the physical structure of the problem, and thus are responsible for the stability of the numerical method. We note that the SSM and the $S U P G$ formulations of the MHD equations have the identical structure except for the value of the stabilization parameters $\tau_{u}$ and $\tau_{B}$. In the SSM, the stabilization parameters $\tau_{u}^{\mathrm{RFB}}$ and $\tau_{B}^{\mathrm{RFB}}$ are explicitly given by

$$
\tau_{u}^{\mathrm{RFB}}=\frac{1}{|K|} \int_{K} b_{K}^{u} \mathrm{~d} K \quad \text { and } \quad \tau_{B}^{\mathrm{RFB}}=\frac{1}{|K|} \int_{K} b_{K}^{B} \mathrm{~d} K
$$

where $b_{K}^{u}$ and $b_{K}^{B}$ are the unique solutions of the following boundary-value problems in a typical element $K$ :

$$
\begin{aligned}
-\varepsilon \triangle b_{K}^{u}+\mathbf{u}_{1}^{n} \cdot \nabla b_{K}^{u}=1 & \text { in } K \\
b_{K}^{u}=0 & \text { on } \partial K
\end{aligned}
$$

and

$$
\begin{aligned}
-\beta \triangle b_{K}^{B}=1 & \text { in } K \\
b_{K}^{B}=0 & \text { on } \partial K
\end{aligned}
$$

Equation (20) above is of convection-diffusion type and therefore the bubble component of the velocity variable has the major influence on the stabilization of the method. 
Note that, finding the exact solutions $b_{K}^{u}$ using (20) and $b_{K}^{B}$ using (21) may not be an easy task in an arbitrary triangular domain. Therefore, a cheap and efficient approximation to $b_{K}^{u}$ and $b_{K}^{B}$ which generates qualitatively the same behavior with the exact function $b_{K}^{u}$ and $b_{K}^{B}$ is required. In that context, we specify a subgrid that consists of three vertices denoted by $V_{i}$ of the triangle plus a single additional node $N$ joined to the three vertices in the interior of each element and approximate the bubble function over the specified subgrid by choosing the location of the additional node such that it gives the best approximation in $L_{1}$ norm. This technique was first proposed by Brezzi et al. in [24] and extended to the Navier-Stokes equations in [25].

The problem (21) is free of convection terms and therefore it is sufficient to take the subgrid node at the triangle's center of gravity. The other problem (20) is more elaborate and the location of the subgrid point is determined using the following two cases: In the first case, where the inflow boundary makes up two edges, let $\mathbf{e}_{2}$ and $\mathbf{e}_{3}$ be two inflow edges (Figure 1). Denote the set of points on the median $V_{1} M$ as a function depending on a single parameter $t: N=(1-t) V_{1}+t M$ where $0<t<1$. Then as the value for $t$, we take

$$
\begin{cases}t=1+\frac{\varepsilon\left(\left|\mathbf{e}_{1}\right|^{2}\right)}{\varepsilon\left(\left|\mathbf{e}_{2}-\mathbf{e}_{3}\right|^{2}-\frac{2}{3}|K|\left(\tilde{\mathbf{u}}_{1}^{n}, \mathbf{v}_{1}\right)\right)} & \text { if } \varepsilon \leqslant \frac{2|K|\left(\tilde{\mathbf{u}}_{1}^{n}, \mathbf{v}_{1}\right) / 3}{3\left|\mathbf{e}_{1}\right|^{2}+\left|\mathbf{e}_{2}-\mathbf{e}_{3}\right|^{2}} \\ t=\frac{2}{3} & \text { otherwise }\end{cases}
$$

where $\tilde{\mathbf{u}}_{1}^{n}$ is the arithmetic average of velocities at the vertices and we denote the length of $\mathbf{e}_{i}$ by $\left|\mathbf{e}_{i}\right|$, the outward unit normal to $\mathbf{e}_{i}$ by $\mathbf{n}_{i}$ and $\boldsymbol{v}_{i}=\left|\mathbf{e}_{i}\right| \mathbf{n}_{i}$ [36]. In the other case where the inflow boundary makes up of a single edge, let $\mathbf{e}_{1}$ be the inflow edge (Figure 1). Then $t$ is taken as

$$
\begin{cases}t=\frac{\varepsilon\left(\left|\mathbf{e}_{2}\right|^{2}+\left|\mathbf{e}_{3}\right|^{2}\right)}{\varepsilon\left(\left|\mathbf{e}_{2}-\mathbf{e}_{3}\right|^{2} / 2-|K|\left(\tilde{\mathbf{u}}_{1}^{n}, \mathbf{v}_{1}\right) / 3\right)} & \text { if } \varepsilon \leqslant \frac{-2|K|\left(\tilde{\mathbf{u}}_{1}^{n}, \mathbf{v}_{1}\right) / 3}{3\left(\left|\mathbf{e}_{2}\right|^{2}+\left|\mathbf{e}_{3}\right|^{2}\right)-\left|\mathbf{e}_{2}-\mathbf{e}_{3}\right|^{2}} \\ t=\frac{2}{3} & \text { otherwise }\end{cases}
$$

Once the location of the subgrid points is determined, a reasonably good approximation to the stabilization parameter $\tau_{u}^{\mathrm{RFB}}$ and $\tau_{B}^{\mathrm{RFB}}$ can be obtained. The approximate values of $\tau_{u}^{\mathrm{RFB}}$ and $\tau_{B}^{\mathrm{RFB}}$

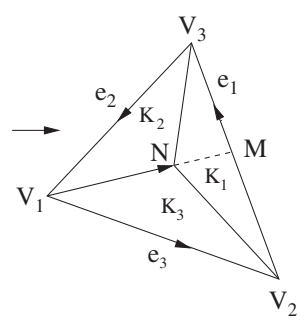

Case 1: Two inflow edges

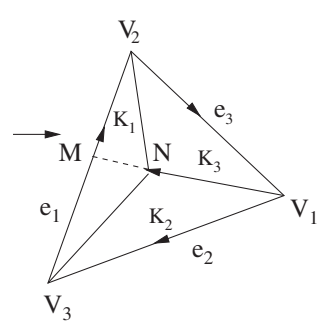

Case 2: One inflow edge

Figure 1. Types of inflow boundaries. 


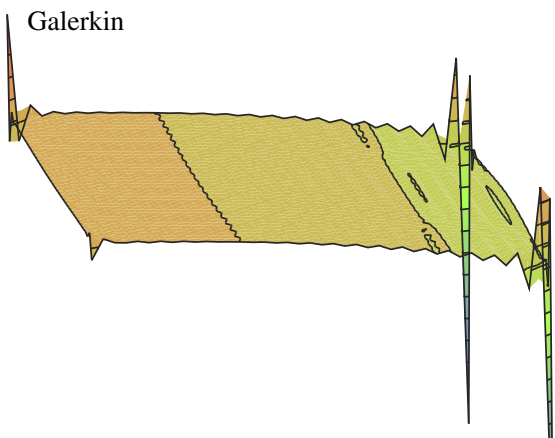

$\mathrm{p}, \min =-19.6073, \max =7.21989$
Exact

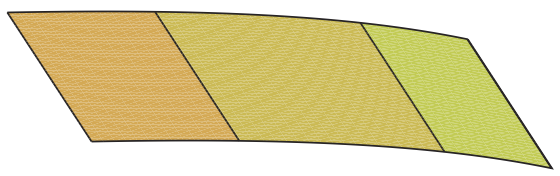

$\mathrm{p}, \min =-2.09726, \max =1.09726$

Figure 2. Pressure elevations obtained from standard Galerkin FEM versus the exact solution.

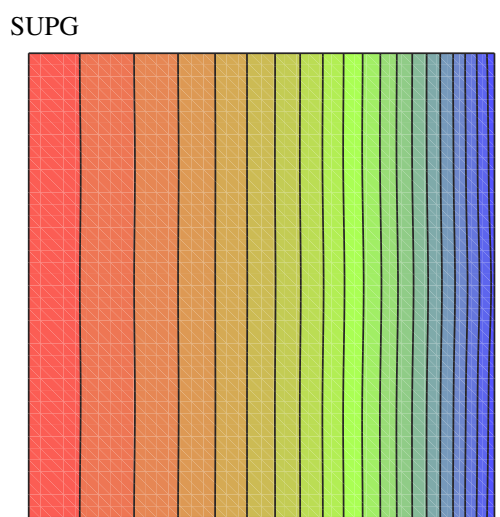

$\mathrm{p}, \min =-2.11672, \max =1.105$

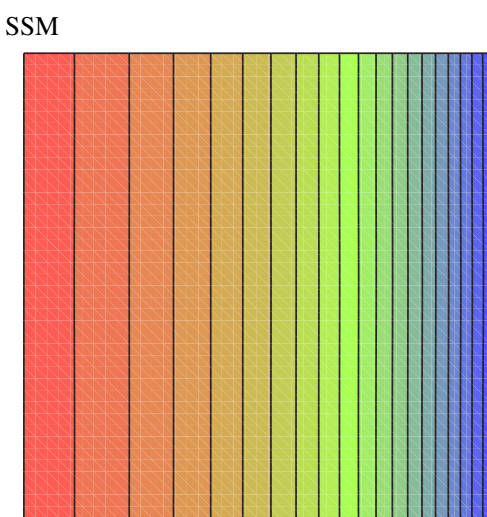

$\mathrm{p}, \min =-2.10967, \max =1.10301$

Exact

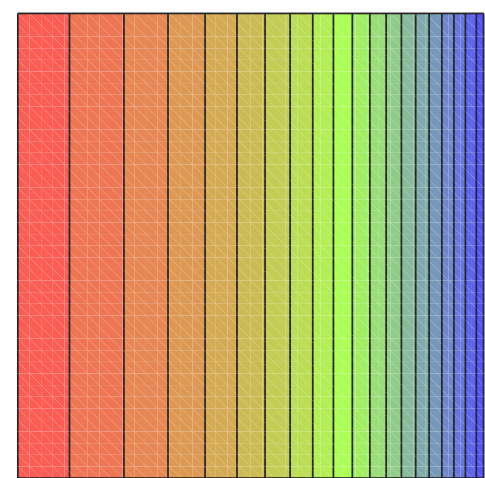

$\mathrm{p}, \min =-2.09726, \max =1.09726$

Figure 3. Pressure contours obtained from the SUPG, the SSM and the exact solutions. 

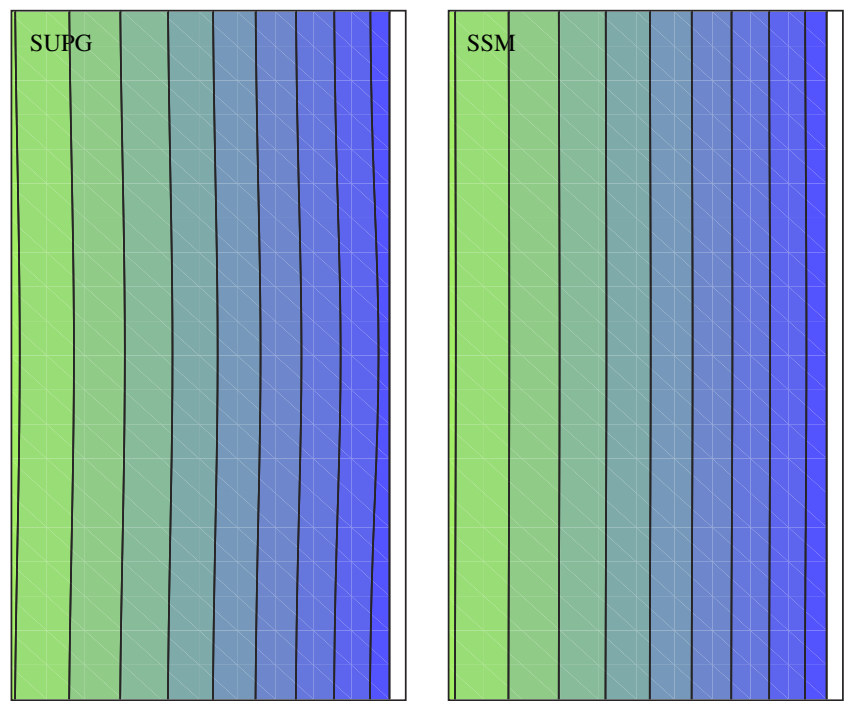

Figure 4. Zoom for the pressure contours obtained from the SUPG and the SSM.

are computed by the following formulas:

$$
\begin{aligned}
& \tilde{\tau}_{u}^{\mathrm{RFB}}=\frac{1}{|K|} \int_{K} b_{K}^{u *}=\frac{1}{|K|} \frac{\left(\int_{K} b_{K}^{u}\right)^{2}}{\varepsilon \int_{K}\left|\nabla b_{K}^{u}\right|^{2}}=\frac{4|K|}{9 \varepsilon \sum_{i}\left|e_{i}\right|^{2} /\left|K_{i}\right|} \\
& \tilde{\tau}_{B}^{\mathrm{RFB}}=\frac{1}{|K|} \int_{K} b_{K}^{B *}=\frac{1}{|K|} \frac{\left(\int_{K} b_{K}^{B}\right)^{2}}{\beta \int_{K}\left|\nabla b_{K}^{B}\right|^{2}}=\frac{4|K|}{9 \beta \sum_{i}\left|e_{i}\right|^{2} /\left|K_{i}\right|}
\end{aligned}
$$

where $K_{i}$ is the area of the $i$ th sub-triangle, $b_{K}^{u *}$ and $b_{K}^{B^{*}}$ are the pseudo-bubble functions that approximate to $b_{K}^{u}$ and $b_{K}^{B}$, respectively [24]. Once we find $\tilde{\tau}_{u}^{\mathrm{RFB}}$ and $\tilde{\tau}_{B}^{\mathrm{RFB}}$, we use them in Equation (18) in place of $\tau_{u}^{\mathrm{RFB}}$ and $\tau_{B}^{\mathrm{RFB}}$.

\section{NUMERICAL RESULTS}

In this section, we present some numerical results obtained by the SSM presented above and compare it with the well-known results in the literature. We work on three problems: (1) a test problem on a unit square with exact solution; (2) MHD flow inside a cavity; and (3) MHD flow over a step. We use the tool Visualization Generale Interactive d'Ecoulements to visualize the numerical results. The iteration cycle that resolves the nonlinearity of the problem stops when the maximum norm of the error is less than $10^{-6}$. 

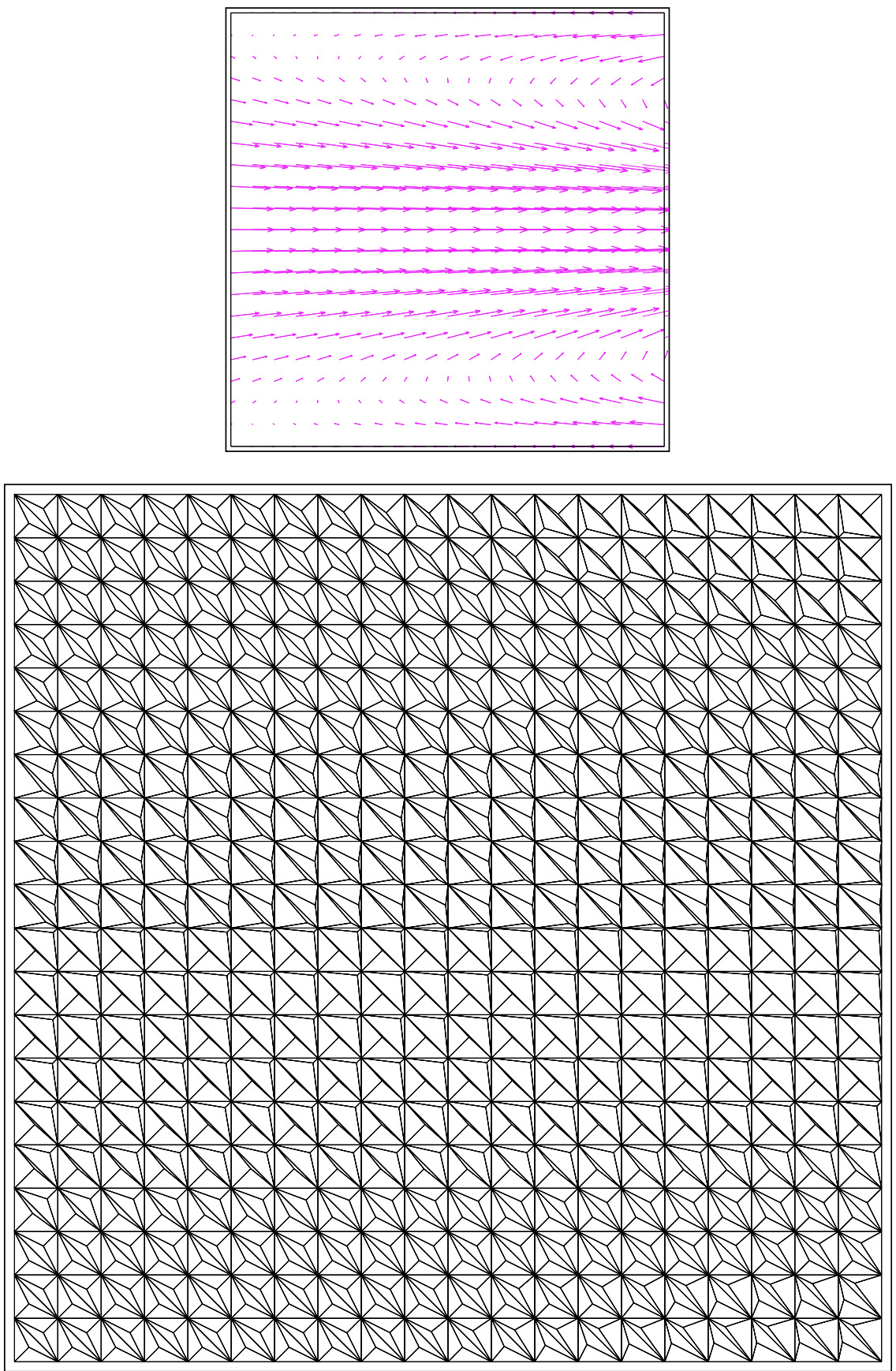

Figure 5. Velocity flow vectors and adaption of the position of the subgrid point. 

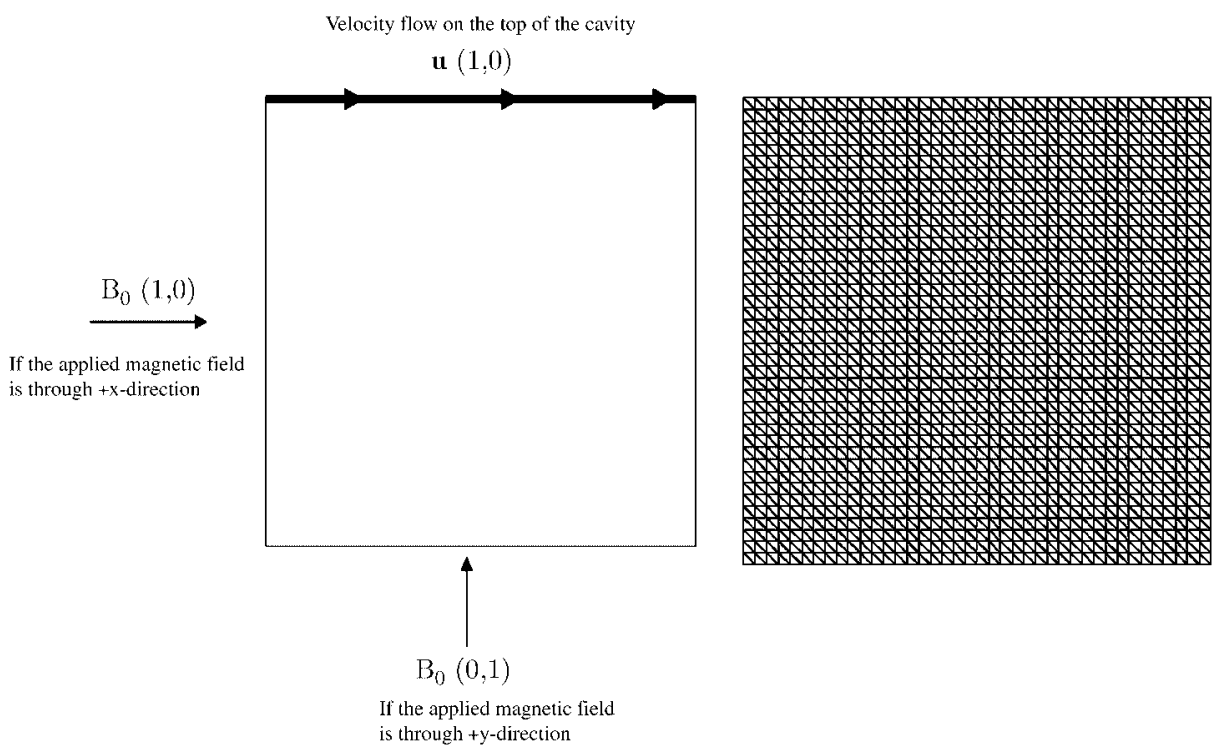

Figure 6. The problem statement and a uniform mesh with 3200 triangular elements used in the approximation of the cavity flow problem.

\subsection{A test problem with the exact solution}

We solve the MHD equations on the following test problem. Boundary conditions are obtained from the exact values of $u_{1}, u_{2}, B_{1}, B_{2}$ and $p$, which satisfy Equations (6)-(10) with $R e=100$, Rem $=10$ and $H a=10$ on the unit square $[0,1] \times[0,1]$ given in [5] as

$$
\begin{aligned}
u_{1} & =1-\exp (x) \cos (2 \pi y) \\
u_{2} & =\exp (x) \sin (2 \pi y) /(2 \pi) \\
B_{1} & =\cos (\pi x) \cos (\pi y) \\
B_{2} & =\sin (\pi x) \sin (\pi y) \\
p & =(1-\exp (2 x)) / 2+C
\end{aligned}
$$

where $C$ is any constant value. We have already noted that the pressure is determined up to a constant. Therefore to satisfy the condition $\int_{\Omega} p=0$ for the pressure, the value of $C$ is selected as $C=1.0972640247327$.

The test problem is first solved by using linear shape functions for all unknowns. In Figure 2 it can be seen that there are oscillations in the pressure values if the standard Galerkin FEM is employed to obtain the approximate solution. The exact solution is smooth though.

In order to obtain stable solutions for the MHD equations, stabilized FEMs should be used. The same test problem is solved by using the SUPG and the SSM methods and obtained solutions are compared with the exact one. We will concentrate on the pressure solutions in which the stabilization is more apparent. 


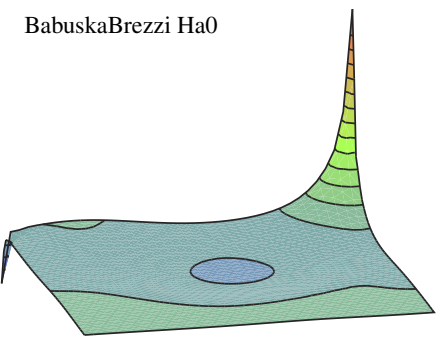

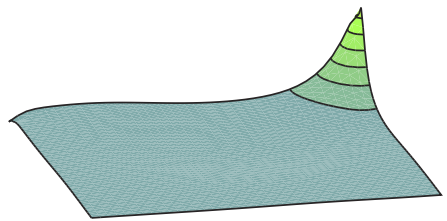

$\mathrm{p}, \min =-0.0590911, \max =0.493543$

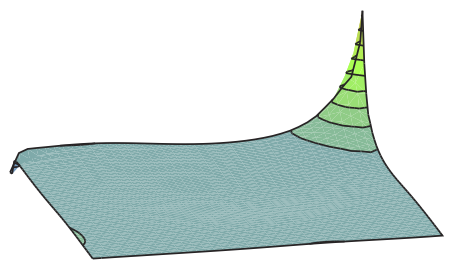

$\mathrm{p}, \min =-0.115918, \max =0.716923$

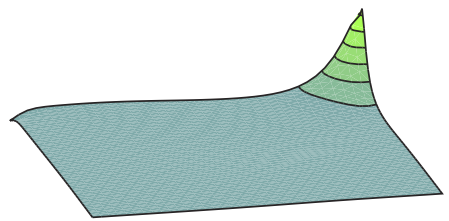

$\mathrm{p}, \min =-0.0671373, \max =0.473249$

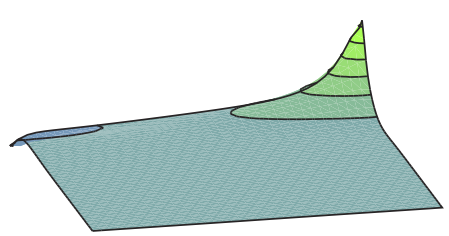

$\mathrm{p}, \min =-0.148306, \max =0.471691$
SSM Ha10

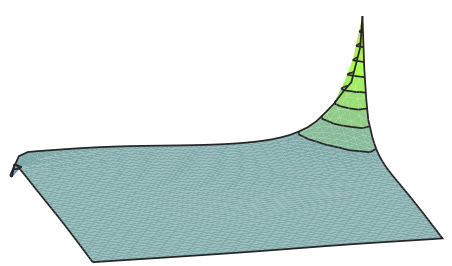

$\mathrm{p}, \min =-0.12463, \max =0.695679$

SSM Ha100

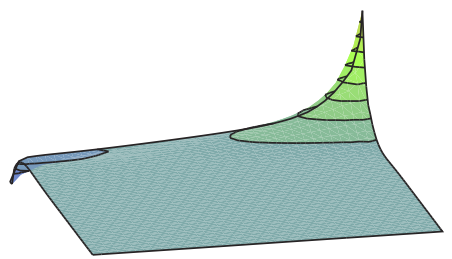

$p, \min =-0.220125, \max =0.670419$ $p, \min =-0.311059, \max =1.17124$

BabuskaBrezzi Ha10

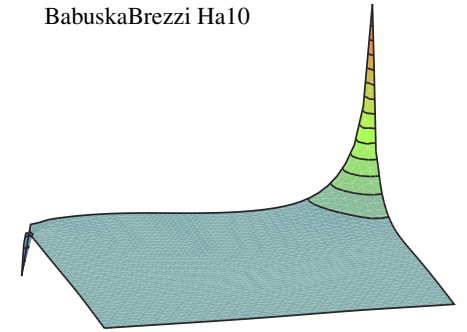

$\mathrm{p}, \min =-0.322675, \max =1.14661$

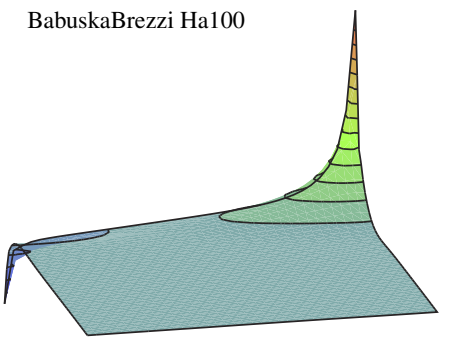

$\mathrm{p}, \min =-0.460975, \max =1.13513$

Figure 7. Pressure elevations obtained from the SUPG, the SSM and the formulation using Babuska-Brezzi condition for $H a=0, H a=10$ and $H a=100$ (applied magnetic field is through $+x$ direction).

Table I. Error of the pressure variable in $L_{2}$ norm.

\begin{tabular}{lcc}
\hline Hartmann number & SSM & SUPG \\
\hline 0 & 0.0057153469 & 0.0072926928 \\
10 & 0.0043141906 & 0.0056119763 \\
100 & 0.0038388398 & 0.0054411338 \\
\hline
\end{tabular}




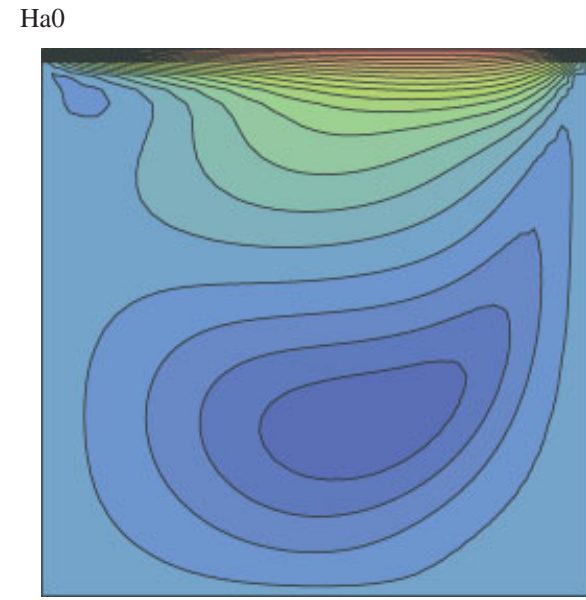

$\mathrm{u} 1, \min =-0.267119, \max =1$
Ha100

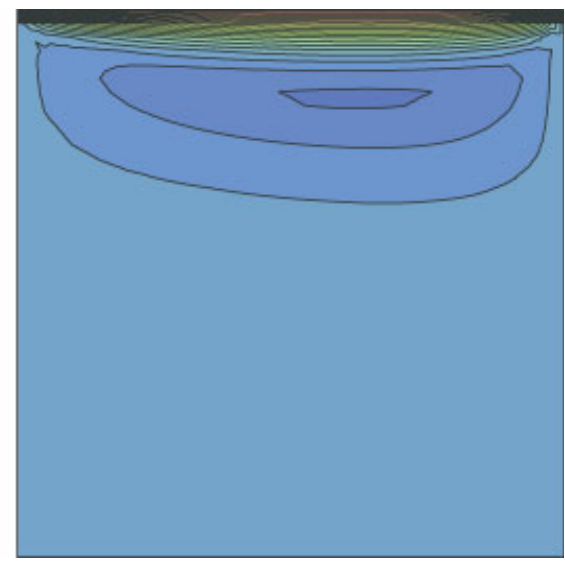

$\mathrm{u} 1, \min =-0.156689, \max =1$

Figure 8. Velocity component $\left(u_{1}\right)$ contours for $H a=0$ and $H a=100$ with the SSM (applied magnetic field is through $+x$ direction).
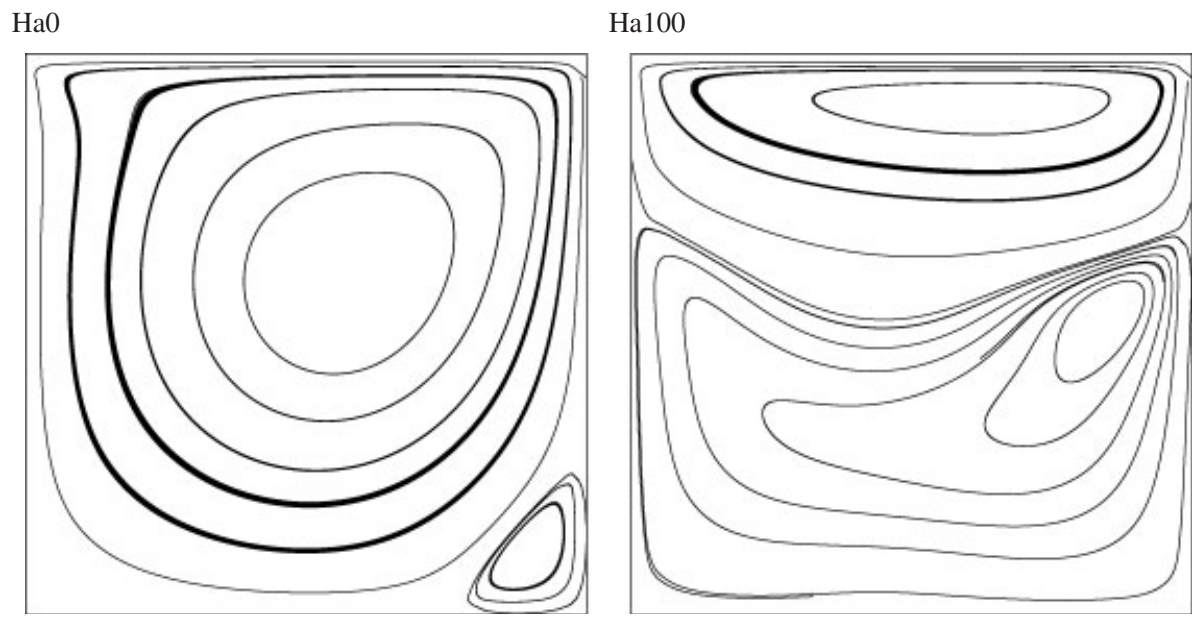

Figure 9. Streamlines of the velocity for $H a=0$ and $H a=100$ with the SSM (applied magnetic field is through $+x$ direction).

It is seen from Figure 3 that the SSM produces more accurate approximations compared with the SUPG method although the stabilization is pronounced in both the methods. The SSM is more effective in the elimination of the disturbances and oscillations in the pressure solution. This can be observed clearly from Figure 4. Notice that the pressure contours obtained from the SSM are smoother than the contours obtained from the SUPG. 
$\mathrm{Ha} 0$

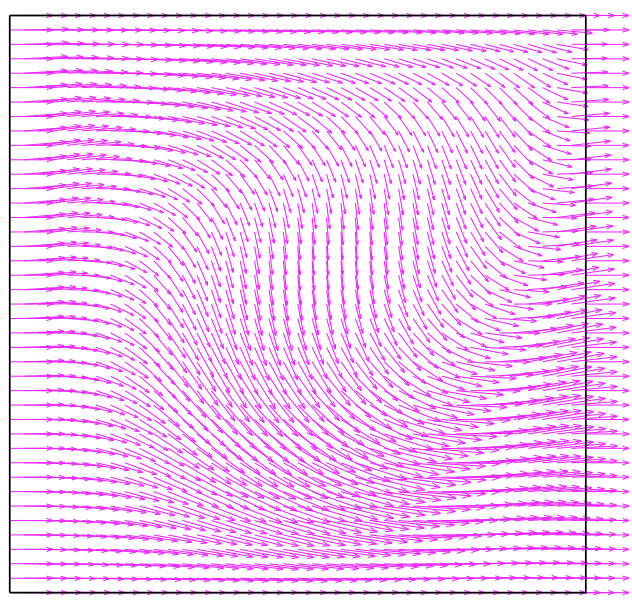

Ha100

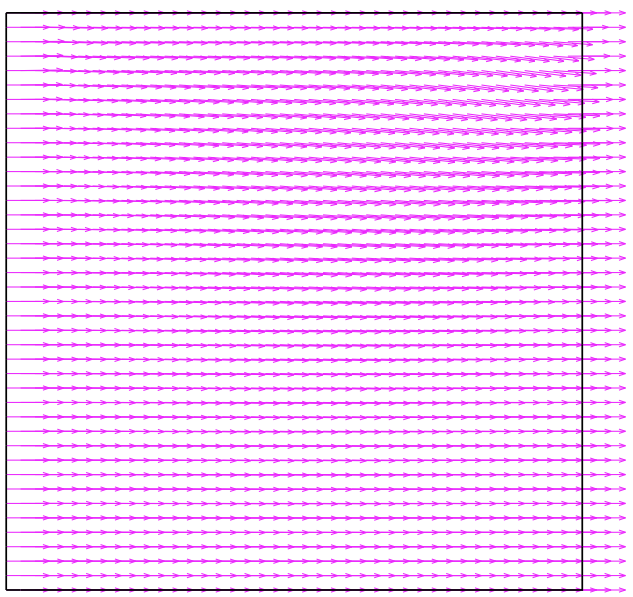

Figure 10. Flow vectors of the magnetic field for $H a=0$ and $H a=100$ with the SSM (applied magnetic field is through $+x$ direction).

The position of the subgrid point is determined by the direction and magnitude of the velocity flow vector during the iteration process. In Figure 5, the resulting velocity flow vector and the location of the subgrid point that plays the main role in the stabilization are displayed over the global mesh. One can observe that in the regions where the magnitude of the velocity is large, the adaptation of the location of the subgrid point is more pronounced. This shows that the location of the subgrid point is critical in the stabilization.

\subsection{Cavity flow}

MHD equations (6)-(10) are solved in a square cavity (Figure 6). Fluid Reynolds number and the magnetic Reynolds numbers are fixed at $R e=400, R e m=40$ in the calculations. The effect of the different values in the Hartmann number $(H a=0,10,100)$ is investigated in the flow under a constant applied magnetic field through $+x$-direction. Dirichlet-type boundary conditions for the magnetic field components are imposed on all over the boundaries as $B_{0}=(1,0)$ when the applied magnetic field is through $+x$-direction and $B_{0}=(0,1)$ when the applied magnetic field is through $+y$-direction. The fluid is moving through $+x$-direction at the top of the cavity with $u_{1}=1$ and $u_{2}=0$ and zero on all the other boundaries.

In Figure 7, pressure elevations for different values of the Hartmann number are displayed for three different methods (the SUPG, the SSM and the formulation satisfying Babuska-Brezzi condition). It can be concluded that the SSM again predicts the solution more accurately compared with the SUPG method and captures the characteristic behaviors of the pressure very well. It is more apparent from the errors of the pressure variable in standard $L_{2}$ norm, given in Table I, that the SSM solution is superior to the SUPG solution. In drawing pressure elevations and in the calculation of $L_{2}$ norms, the formulation satisfying Babuska-Brezzi condition is taken as a reference solution. The existence of the boundary layer formation, which is the 


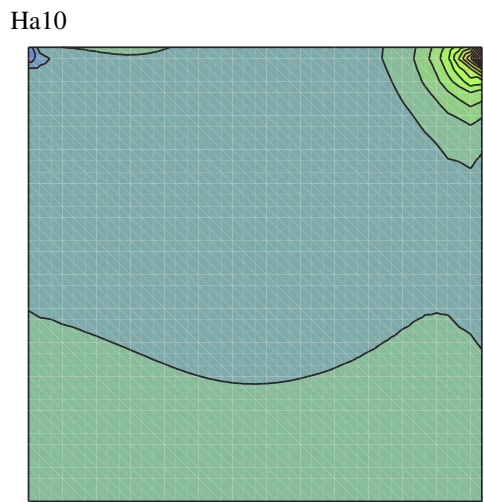

$\mathrm{p}, \min =-0.126982, \max =0.684394$

\section{Ha10}

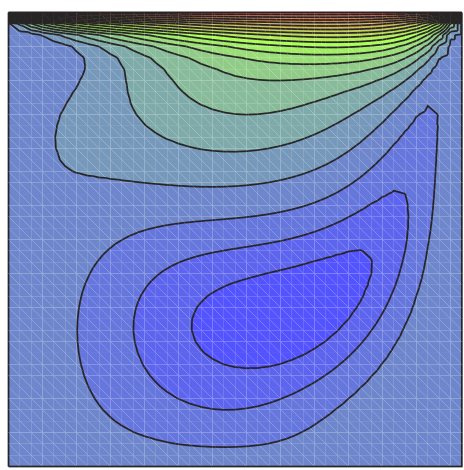

$\mathrm{u} 1, \min =-0.225696, \max =1$

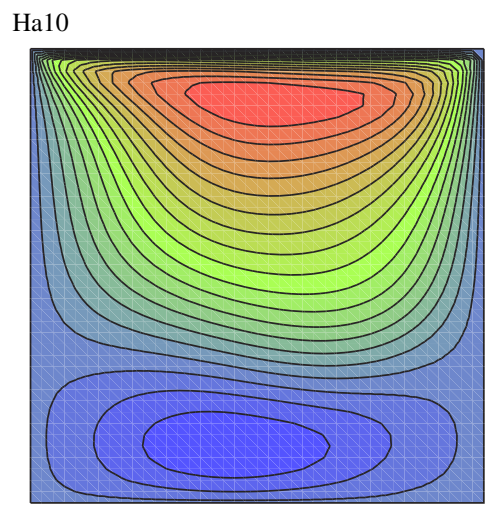

$\mathrm{B} 1, \min =-0.191294, \max =1.01707$

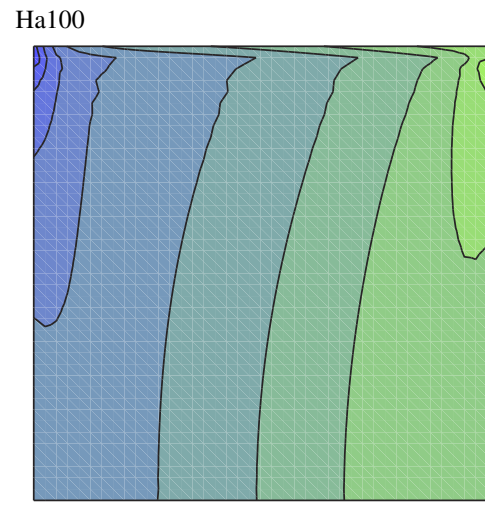

$p, \min =-0.280725, \max =0.200934$

\section{Ha100}

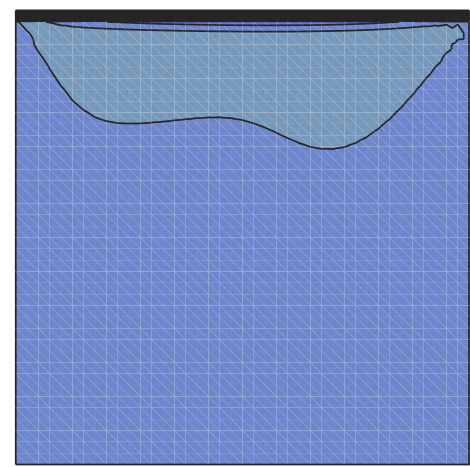

$\mathrm{u} 1, \min =-0.0864218, \max =1$

\section{Ha100}

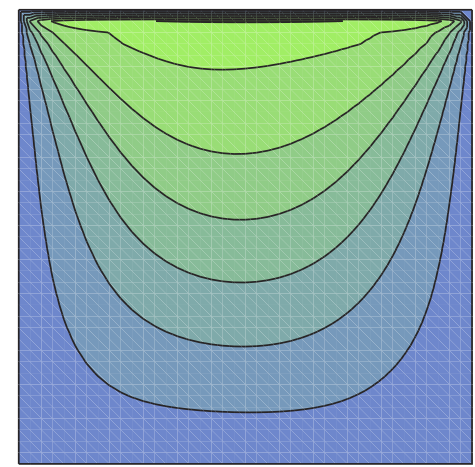

B1, $\min =0, \max =0.418229$

Figure 11. Pressure, the first component of the velocity and the magnetic field contours for $H a=10$ and $H a=100$ with the SSM (applied magnetic field is through $+y$ direction). 


\section{Ha100}

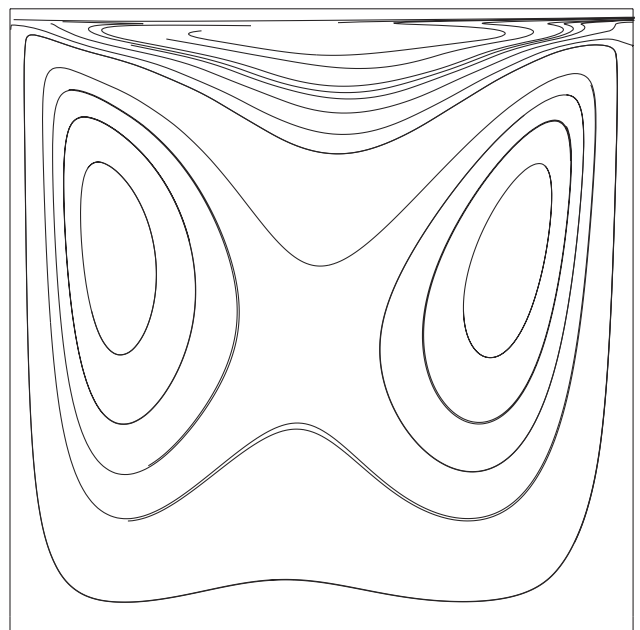

Ha100

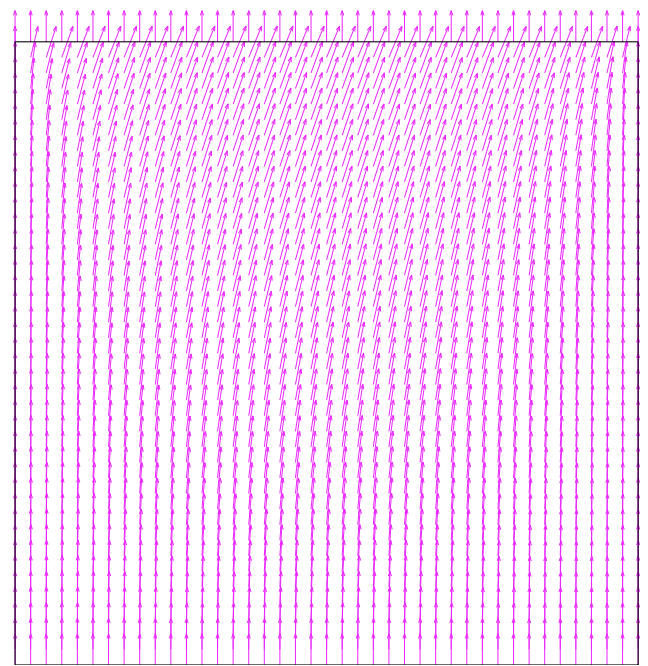

Figure 12. Streamlines for the velocity and flow vectors of the magnetic field for $H a=100$ with the SSM (applied magnetic field is through $+y$ direction).
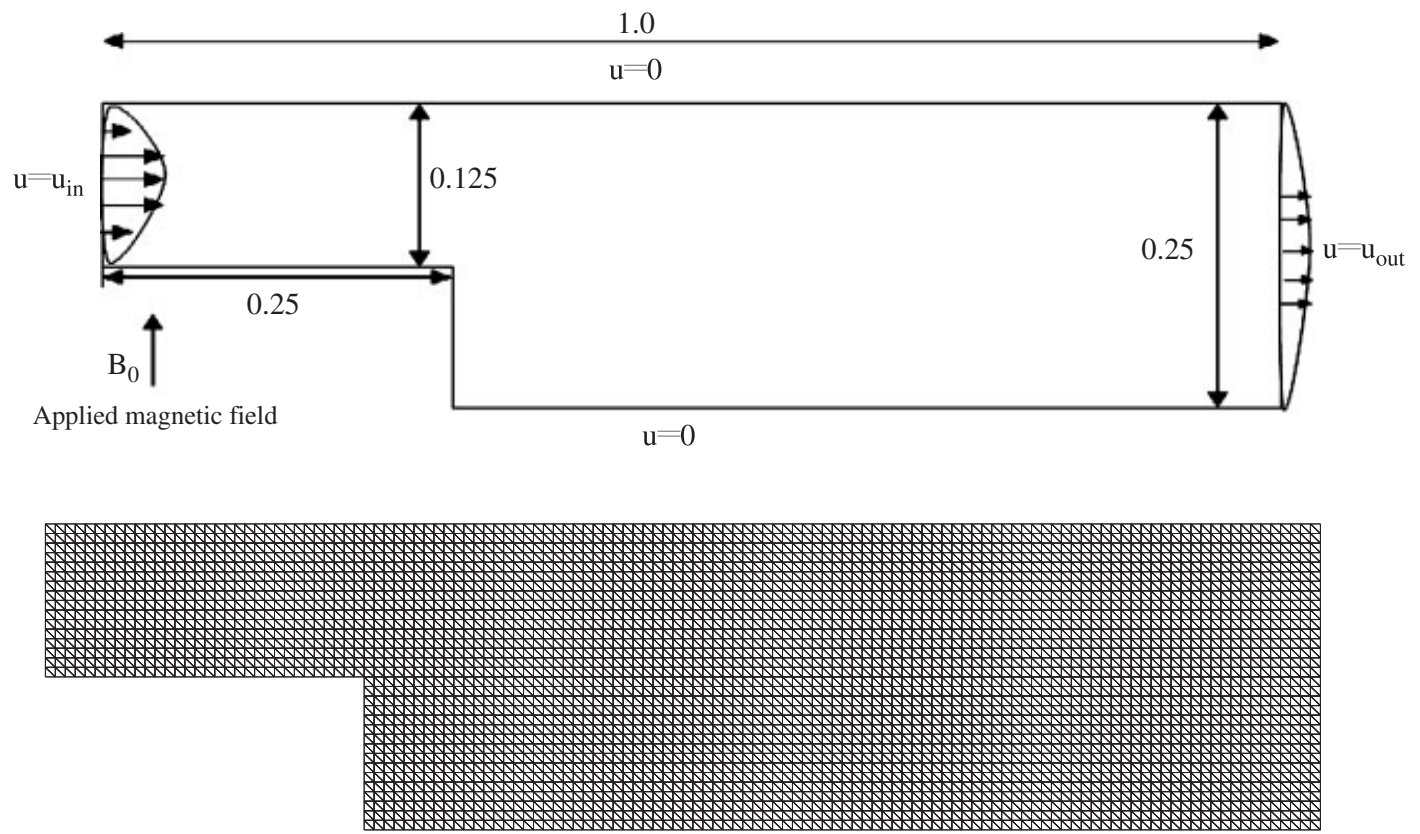

Figure 13. The problem statement and a uniform mesh with 7168 triangular elements used in the approximation. 
H0

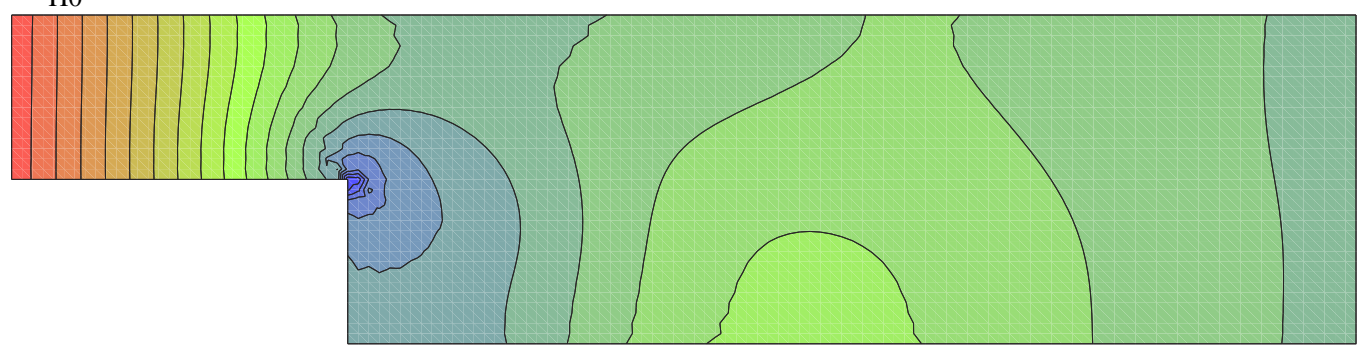

H5
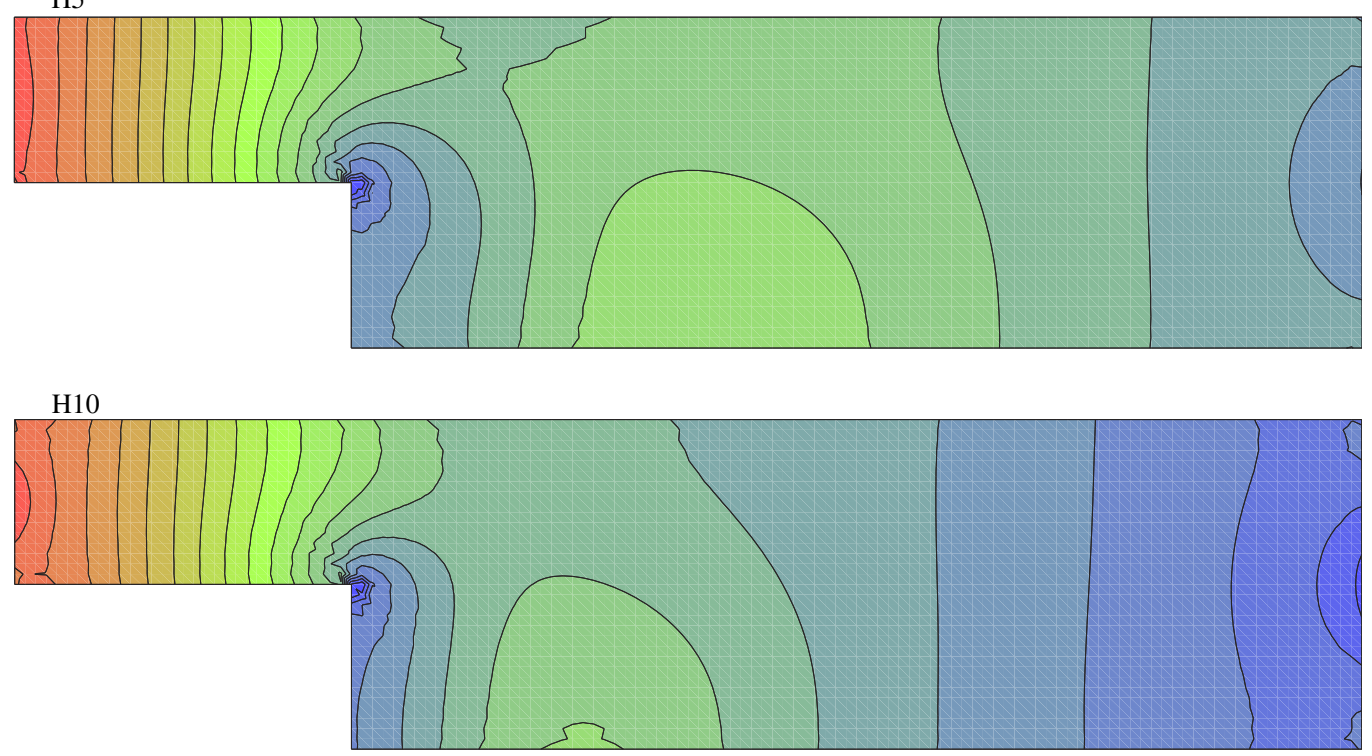

Figure 14. Pressure contours for $H a=0, H a=5$ and $H a=10$.

well-known behavior of the MHD flows, is displayed in Figure 8 in terms of the first component of the velocity as Hartmann number increases. Streamlines for the velocity and flow vectors for the magnetic field are also displayed in Figures 9 and 10, respectively. Obtained solutions, which are compatible with the solutions in the literature, show the validity of the proposed method.

The cavity flow problem is also solved with the SSM when the magnetic field is applied through $+y$-direction for the same fluid Reynolds number and magnetic Reynolds number and Hartmann numbers 10 and 100. In Figure 11, it is seen that as Hartmann number increases, pressure contours are getting perpendicular to the $y$-axis and boundary layer formation in the velocity and in the magnetic field are pronounced more clearly. The same behavior is observed from the streamlines of the velocity and flow vectors of the magnetic field in Figure 12. We also note that the solutions presented here show consistency with the numerical results in the literature $[6,16,22]$. 

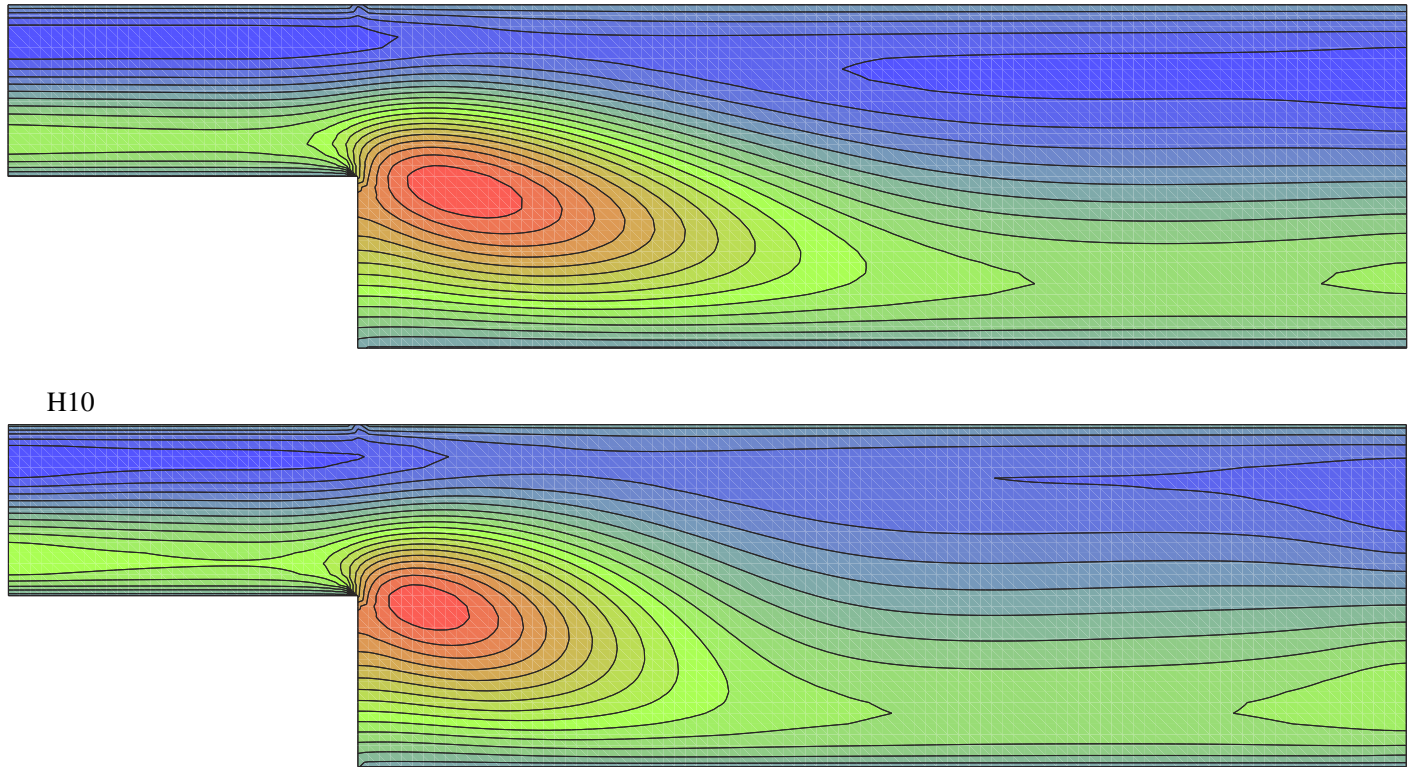

Figure 15. The contour plot of the $x$-component of the magnetic field $B$ for $H a=5$ and $H a=10$.

\subsection{MHD flow over a step}

This is another standard benchmark problem. The statement of the problem and the mesh is given in Figure 13. The flow of the fluid is through $+x$-direction and applied magnetic field is through $+y$ direction. The walls of the pipe are considered perfect conductors. The velocity is prescribed at the inlet and the outlet to a Poiseuille profile such that $\mathbf{u}_{\text {out }}=\frac{1}{2} \mathbf{u}_{\text {in }}$. The problem is solved with the SSM for $R e=100$, Rem $=1$ and Hartmann numbers 0, 5 and 10 .

Figures 14 and 15 show the magnetic field effect in the pressure and in the $x$-component of the magnetic field as Hartmann number increases, respectively. It is displayed in terms of streamlines in Figure 16 such that the recirculation after the step decreases as Hartmann number increases. Obtained solutions are in a good agreement with the other solutions in the literature [20,23].

\section{CONCLUSION}

We consider a SSM for the approximate solution of the incompressible MHD equations in the framework of the TLFEM. The domain is planar and the discretization is triangular. The presentation above shows that the TLFEM with the stabilizing subgrid produces stable and accurate approximations in a variety of problem configurations establishing that it is applicable to nonlinear 

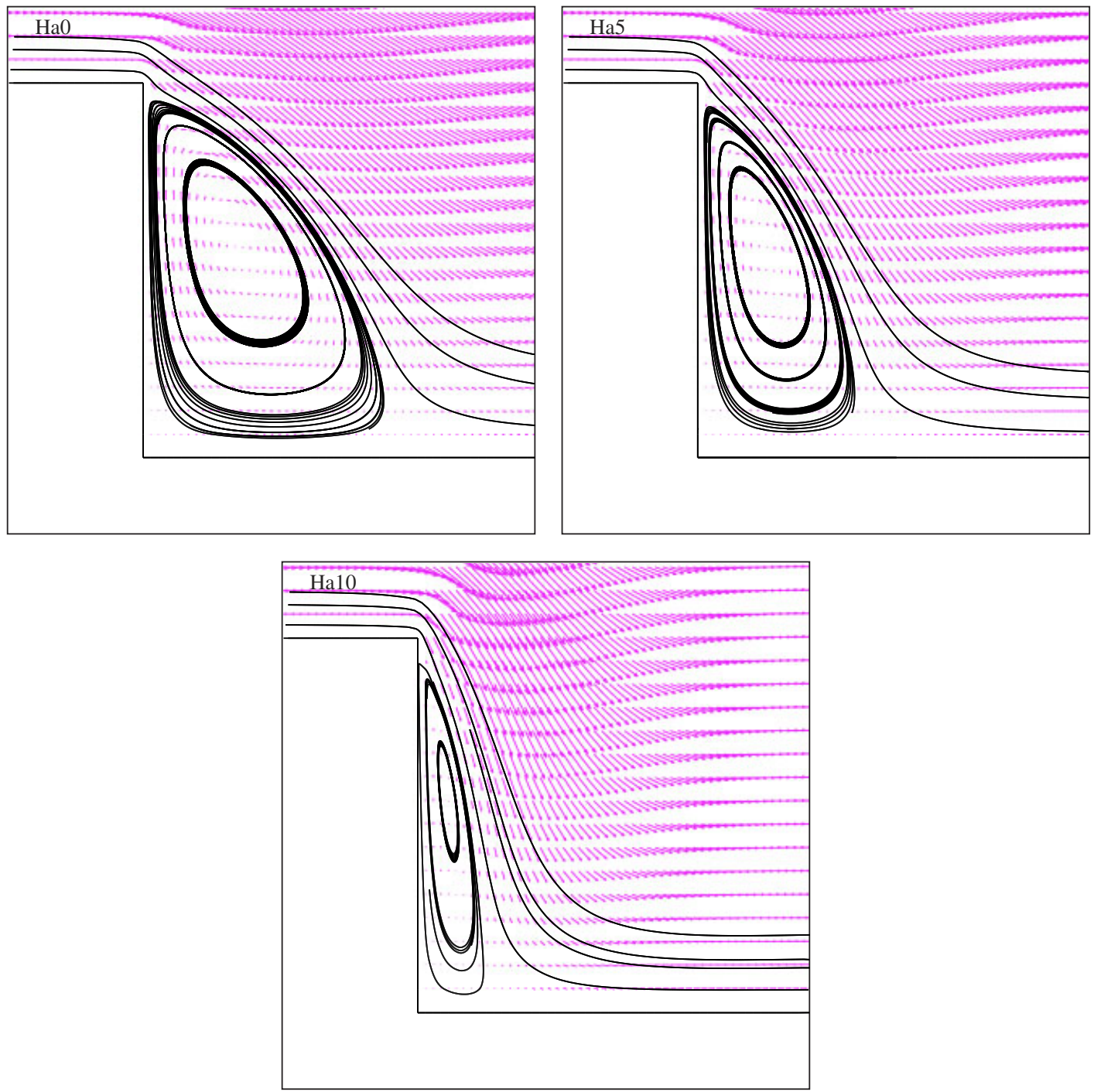

Figure 16. Velocity flow vectors and streamlines zoom in behind the step for $H a=0, H a=5$ and $H a=10$.

problems in two-dimension. Numerical experiments further indicate that the proper choice of the subgrid node may play a significant role in obtaining more accurate approximations, especially for high Reynolds and moderate Hartmann numbers.

\section{ACKNOWLEDGEMENTS}

This work is supported by The Scientific and Technical Research Council of Turkey (TUBITAK) (Contract \# 105T091). 


\section{REFERENCES}

1. Shercliff JA. Steady motion of conducting fluids in pipes under transverse magnetic fields. Proceedings of the Cambridge Philosophical Society 1953; 49:136-144.

2. Dragos L. Magnetofluid Dynamics. Abacus Press: Tunbridge Wells, U.K., 1975.

3. Krzeminski SK, Cala A, Smialek M. Numerical simulation of 2D MHD flows $\Psi-\xi-\mathbf{A}$ method. IEEE Transactions on Magnetics 1996; 32(3):990-993.

4. Sekhar TVS, Ravikumar TVR, Kumar H. MHD flow past a sphere at low and moderate Reynolds numbers. Computational Mechanics 2003; 31:437-444.

5. Sheu TWH, Lin RK. Development of a convection-diffusion-reaction magnetohydrodynamic solver on a nonstaggered grids. International Journal for Numerical Methods in Fluids 2004; 45:1209-1233.

6. Navarro HA, Cabezas-Gómez L, César da Silva R, Montagnoli AN. A generalized alternating-direction implicit scheme for incompressible magnetohydrodynamic viscous flows at low magnetic Reynolds number. Applied Mathematics and Computation 2007; 189:1601-1613.

7. Kumamaru H, Kodama S, Hirano H, Itoh K. Three-dimensional numerical calculations on liquid-metal magnetohydrodynamic flow in magnetic-field inlet-region. Journal of Nuclear Science and Technology 2004; 41(5):624-631.

8. Wiedmer M. Finite element approximation for equations of magnetohydrodynamics. Mathematics of Computation 1999; 69(229):83-101.

9. Gunzburger MD, Meir AJ, Peterson JS. On the existence, uniqueness, and finite element approximation of solutions of the equations of stationary, incompressible magnetohydrodynamics. Mathematics of Computation 1991; 56(194):523-563.

10. Meir AJ, Schmidt PG. Analysis and numerical approximation of a stationary MHD flow problem with non ideal boundary. SIAM Journal on Numerical Analysis 1999; 36(4):1304-1332.

11. Schötzau D. Mixed finite element methods for stationary incompressible magneto-hydrodynamics. Numerische Mathematik 2004; 96:771-800.

12. Hasler U, Schneebeli A, Schötzau D. Mixed finite element approximation of incompressible MHD problems based of weighted regularization. Applied Numerical Mathematics 2004; 51:19-45.

13. Layton WJ, Meir AJ, Schmidt PG. A two-level discretization method for the stationary MHD equations. Electronic Transactions of Numerical Analysis 1997; 6:198-210.

14. Verardi SLL, Cardoso JR. A solution of two-dimensional magnetohydrodynamic flow using the finite element method. IEEE Transactions on Magnetics 1998; 34(5):3134-3137.

15. Verardi SLL, Cardoso JR. Three-dimensional finite element analysis of MHD duct flow by the penalty function formulation. IEEE Transactions on Magnetics 2001; 37(5):3384-3387.

16. Krzemiński SK, Śmialek M, Wlodarczyk M. Finite element approximation of biharmonic mathematical model for MHD flow using $\Psi$-An approach. IEEE Transactions on Magnetics 2000; 36(4):1313-1318.

17. Tezer-Sezgin M, Koksal S. FEM for solving MHD flow in a rectangular duct. International Journal for Numerical Methods in Engineering 1989; 28:445-459.

18. Nesliturk AI, Tezer-Sezgin M. The finite element method for MHD flow at high Hartmann numbers. Computer Methods in Applied Mechanics and Engineering 2005; 194:1201-1224.

19. Nesliturk AI, Tezer-Sezgin M. Finite element method solution of electrically driven magnetohydrodynamic flow. Journal of Computational and Applied Mathematics 2006; 192:339-352.

20. Gerbeau JF. A stabilized finite element method for the incompressible magnetohydrodynamic equations. Numerische Mathematik 2000; 87:83-111.

21. Salah NB, Soulaimani A, Habashi WG, Fortin M. A conservative stabilized finite element method for the magneto-hydrodynamic equations. International Journal for Numerical Methods in Fluids 1999; 29:535-554.

22. Salah NB, Soulaimani A, Habashi WG. A finite element method for magnetohydrodynamics. Computer Methods in Applied Mechanics and Engineering 2001; 190:5867-5892.

23. Codina R, Silva NH. Stabilized finite element approximation of the stationary magneto-hydrodynamics equations. Computational Mechanics 2006; 38:344-355.

24. Brezzi F, Marini D, Russo A. On the choice of a stabilizing subgrid for convection-diffusion problems. Computer Methods in Applied Mechanics and Engineering 2005; 194:127-148.

25. Nesliturk AI, Aydin SH, Tezer-Sezgin M. Two-level finite element method with a stabilizing subgrid for the incompressible Navier-Stokes equations. International Journal for Numerical Methods in Fluids 2007; 58: $551-572$.

Copyright (C) 2009 John Wiley \& Sons, Ltd.

Int. J. Numer. Meth. Fluids 2010; 62:188-210

DOI: $10.1002 / f l d$ 
26. Brezzi F. On the existence, uniqueness and approximation of saddle-point problems arising from Lagrange multipliers. RAIRO_Mathematical Modelling and Numerical Analysis 1974; 8:129-151.

27. Babuska I. The finite element method with Lagrangian multipliers. Numerische Mathematik 1973; 20:179-192.

28. Brooks AN, Hughes TJR. Streamline upwind/Petrov-Galerkin formulations for convection dominated flows with particular emphasis on the incompressible Navier-Stokes equations. Computer Methods in Applied Mechanics and Engineering 1982; 32:199-259.

29. Johnson C, Nävert U, Pitkäranta J. Finite element methods for linear hyperbolic problem. Computer Methods in Applied Mechanics and Engineering 1984; 45:285-312.

30. Brezzi FF, Bristeau MO, Franca LP, Mallet M, Rogé G. A relationship between stabilized finite element methods and the Galerkin method with bubble functions. Computer Methods in Applied Mechanics and Engineering 1992; 96:117-129.

31. Franca LP, Farhat C. Bubble functions prompt unusual stabilized finite element methods. Computer Methods in Applied Mechanics and Engineering 1995; 123:299-308.

32. Franca LP, Macedo AP. A two-level finite element method and its application to the Helmholtz equation. International Journal for Numerical Methods in Engineering 1998; 43:23-32.

33. Brezzi F, Hughes TJR, Marini LD, Russo A, Suli E. A priori error analysis of residual-free bubbles for advection-diffusion problems. SIAM Journal on Numerical Analysis 1999; 36:1933-1948.

34. Sangalli G. Global and local error analysis for the residual-free bubbles method applied to advection-dominated problems. SIAM Journal on Numerical Analysis 2000; 38:1496-1522.

35. Russo A. Bubble stabilization of finite element methods for the linearized incompressible Navier-Stokes equations. Computer Methods in Applied Mechanics and Engineering 1996; 132:335-343.

36. Nesliturk AI. A stabilizing subgrid for convection-diffusion problem. Mathematical Models and Methods in Applied Sciences 2006; 16(2):211-231. 